\title{
The magnetic field configuration of a solar prominence inferred from spectropolarimetric observations in the He I $10830 \AA$ triplet ${ }^{\star}$
}

\author{
D. Orozco Suárez ${ }^{1,2}$, A. Asensio Ramos ${ }^{1,2}$, and J. Trujillo Bueno ${ }^{1,2,3}$ \\ 1 Instituto de Astrofísica de Canarias, 38205 La Laguna, Tenerife, Spain \\ e-mail: dorozco@iac.es \\ 2 Departamento de Astrofísica, Universidad de La Laguna, 38206 La Laguna, Tenerife, Spain \\ 3 Consejo Superior de Investigaciones Científicas, 28037 Madrid, Spain
}

Received 23 October 2013 / Accepted 28 March 2014

\begin{abstract}
Context. Determining the magnetic field vector in quiescent solar prominences is possible by interpreting the Hanle and Zeeman effects in spectral lines. However, observational measurements are scarce and lack high spatial resolution.

Aims. We determine the magnetic field vector configuration along a quiescent solar prominence by interpreting spectropolarimetric measurements in the He I $1083.0 \mathrm{~nm}$ triplet obtained with the Tenerife Infrared Polarimeter installed at the German Vacuum Tower Telescope of the Observatorio del Teide.

Methods. The He I $1083.0 \mathrm{~nm}$ triplet Stokes profiles were analyzed with an inversion code that takes the physics responsible for the polarization signals in this triplet into account. The results are put into a solar context with the help of extreme ultraviolet observations taken with the Solar Dynamic Observatory and the Solar Terrestrial Relations Observatory satellites.

Results. For the most probable magnetic field vector configuration, the analysis depicts a mean field strength of 7 gauss. We do not find local variations in the field strength except that the field is, on average, lower in the prominence body than in the prominence feet, where the field strength reaches $\sim 25$ gauss. The averaged magnetic field inclination with respect to the local vertical is $\sim 77^{\circ}$. The acute angle of the magnetic field vector with the prominence main axis is $24^{\circ}$ for the sinistral chirality case and $58^{\circ}$ for the dextral chirality. These inferences are in rough agreement with previous results obtained from the analysis of data acquired with lower spatial resolutions.
\end{abstract}

Key words. Sun: chromosphere - Sun: filaments, prominences

\section{Introduction}

Although the first photographic plates of prominences were taken more than 150 years ago, it took 100 years to discover, by means of the first spectropolarimetric measurements in prominences, that these solar structures are clear manifestations of the confinement of plasma within giant magnetic structures ${ }^{1}$. Prominences, also referred to as filaments when observed against the solar disk, are cool, dense, magnetized formations of $10^{4} \mathrm{~K}$ plasma embedded in the $10^{6} \mathrm{~K}$ solar corona (for reviews see Mackay et al. 2010; Labrosse et al. 2010). They are located above polarity inversion lines (PILs or filament channels), i.e., the line that divides regions of opposite magnetic flux in the photosphere. Morphologically speaking, prominences can be separated into different classes (Pettit 1943; Tandberg-Hanssen 1995). Among them, quiescent prominences are seen as large sheet-like structures suspended above the solar surface and against gravity. The overall structure of quiescent prominences changes little with time, preserving their shape during days and even weeks. Locally, they consist of fine and vertically oriented plasma structures, so-called threads, that evolve continually (e.g., Engvold 1976; Zirker et al. 1994). Recent ob-

\footnotetext{
* A movie is available in electronic form at http: //www . aanda.org

1 There are many papers about solar prominences, but we recommend that the reader first go through the historical work of Einar Tandberg-Hanssen (e.g., Tandberg-Hanssen 1998, 2011).
}

servations taken with the Hinode satellite have revolutionized our knowledge of the fine-scale structuring and dynamics of quiescent prominences; for instance, plasma oscillations, supersonic downflows, or plasma instabilities like the Rayleigh-Taylor instability in prominence bubbles (Berger et al. 2008, 2010; Chae et al. 2008; Okamoto et al. 2007).

The magnetic configuration of quiescent prominences has been investigated first by using the longitudinal Zeeman effect and later by measuring the full Stokes vector in spectral lines sensitive to the joint action of the Zeeman and Hanle effect (e.g., Leroy 1989; and López Ariste \& Aulanier 2007, for reviews). For instance, spectropolarimetric observations in the He I D3 multiplet at $587.6 \mathrm{~nm}$ have greatly contributed to the understanding of the magnetic field configuration in prominences (Athay et al. 1983; Querfeld et al. 1985; Casini et al. 2003). Full Stokes polarimetry in the He I D3 multiplet at $587.6 \mathrm{~nm}$ is accessible from several ground-based observatories, such as the French-Italian telescope (THEMIS) at the Observatorio del Teide, the Advanced Stokes Polarimeter at the Dunn Solar Telescope in Sacramento Peak, or the Istituto Ricerche Solari (IRSOL) observatory.

Of particular interest for infering the magnetic field vector in prominences is the He I triplet at $1083.0 \mathrm{~nm}$. This spectral line can be clearly seen in emission in off-limb prominences (e.g., Merenda et al. 2006) and in absorption in on-disk filaments (e.g., Lin et al. 1998). The He I triplet is sensitive to the joint action of atomic level polarization (i.e., population 
imbalances and quantum coherence among the level's sublevels, generated by anisotropic radiation pumping) and the Hanle (modification of the atomic level polarization due to the presence of a magnetic field) and Zeeman effects (Trujillo Bueno et al. 2002; Trujillo Bueno \& Asensio Ramos 2007). This fact makes the He I $1083.0 \mathrm{~nm}$ triplet sensitive to a wide range of field strengths from $\mathrm{dG}$ (Hanle) to $\mathrm{kG}$ (Zeeman). Important is that the He I $1083.0 \mathrm{~nm}$ triplet is easily observable with the Tenerife Infrared Polarimeter (TIP-II; Collados et al. 2007) installed at the German Vacuum Tower Telescope (VTT) of the Observatorio del Teide (Tenerife, Spain). Finally, an userfriendly diagnostic tool called "HAZEL" (from HAnle and ZEeman Light) is available for modeling and interpreting the He I $1083.0 \mathrm{~nm}$ triplet polarization signals, easing the determination of the strength, inclination and azimuth of the magnetic field vector in many solar structures (Asensio Ramos et al. 2008). The HAZEL code has already been used to analyze He I $1083.0 \mathrm{~nm}$ triplet spectropolarimetric data of prominences (Orozco Suárez et al. 2013), spicules (Centeno et al. 2010; Martínez González et al. 2012), sunspot's super-penumbral fibrils (Schad et al. 2013), emerging flux regions (Asensio Ramos \& Trujillo Bueno 2010), and the quiet solar chromosphere (Asensio Ramos \& Trujillo Bueno 2009). The HAZEL code has also been applied to He I D3 observations of prominences and spicules (Ramelli et al. 2011).

However, the information we have about the spatial variations of the magnetic field vector in solar prominences is still very limited because of the insufficient spatial resolution of the observations, restricted to single point measurements (e.g., Leroy et al. 1983; Athay et al. 1983), single slit measurements (e.g., Merenda et al. 2006), or two-dimensional slit scans (e.g., Casini et al. 2003; Merenda et al. 2007) at spatial resolutions of about $2^{\prime \prime}$, much lower than the subarcseconds resolutions achieved by the Hinode spacecraft in prominence broad-band imaging. We have an approximate picture of the global magnetic properties of quiescent solar prominences, mainly thanks to the information encoded in spectral lines sensitive to the Hanle and Zeeman effect.

The magnetic field in quiescent prominences is fairly uniform and has mean field strengths of tens of gauss, typically in the range of $3 \mathrm{G}$ to $30 \mathrm{G}$. The magnetic field vector forms an acute angle of about $35^{\circ}$ with the prominence long axis (Tandberg-Hanssen \& Anzer 1970; Leroy et al. 1983; Bommier et al. 1994; Casini et al. 2003). The field lines are found to be highly inclined with respect to the local vertical (e.g., Athay et al. 1983). For instance, Leroy et al. (1983) found a mean inclination of $60^{\circ}$ from the local vertical in a sampling of 15 prominences. Their data were limited to single point measurements, and their estimated rms error was about $15^{\circ}$.

More recently, Casini et al. $(2003,2005)$ inferred the vector field map in a quiescent prominence and found inclinations of about $90^{\circ}$ with respect to the local vertical. These authors also report that the field can be organized in patches where it increases locally up to $80 \mathrm{G}$. The magnetic configuration seems to be different for polar crown prominences where the field is found to be inclined by about $25^{\circ}$ with respect to the solar radius vector through the observed point (Merenda et al. 2006). Finally, it has been found that, for $75 \%$ of the analyzed prominences, the perpendicular component of the magnetic field vector to the prominence long axis, or PIL, points to the opposite direction with respect to the photospheric magnetic field. In this case, they are classified as inverse polarity prominences (Leroy et al. 1983).

The magnetic field vector we infer through interpreting polarizations signals, such as those of the He I $1083.0 \mathrm{~nm}$ multiplet, is associated with the coolest and densest prominence material. For this reason, the magnetic field in prominences has also been investigated by indirect means, i.e., by constructing models of the field geometry in order to capture the observed prominence shape and properties (Aulanier \& Demoulin 1998; Aulanier et al. 1999, 1998; Dudík et al. 2012). Such studies have contributed to our present picture of the global magnetic field structure associated to the prominence, although most models assume that the prominence material is suspended in magnetic dips. Among these models, we have the sheared-arcade models (van Ballegooijen \& Martens 1989) and the twisted flux-rope models (Rust \& Kumar 1994). In the first ones, a helical magnetic structure is generated via photospheric shear flow motions that give rise to magnetic reconnection of pre-existing magnetic fields lines near the PIL. The cool material of the prominence is then supported by the magnetic dips of the helical structure via a magnetic tension force (Kippenhahn \& Schlüter 1957; Low \& Petrie 2005; Chae 2010), by MHD-waves pressure (Pécseli \& Engvold 2000), or by the presence of tangled magnetic fields on very small scales (van Ballegooijen \& Cranmer 2010). The twisted flux-rope models suggest that the helical magnetic field structure supporting the prominence material has emerged from below the photosphere. Both models yield magnetic properties compatible with the present, low-resolution observational constraints. The local magnetic field in prominences has also been investigated by interpreting the dynamics of rising plumes using magnetohydrodynamic models (Hillier et al. 2012a,b).

Here we present the results of the analysis of ground-based spectropolarimetric observations of the He I $1083.0 \mathrm{~nm}$ triplet taken in a quiescent solar prominence. The data were obtained with the TIP-II instrument (Collados et al. 2007) installed at the German VTT at the Observatorio del Teide. This instrument is providing observations of solar prominences at spatial resolutions of about $1^{\prime \prime}-1$ '.5 during regular observing conditions, and even below one arcsecond in periods of excellent seeing conditions. In this paper, some of these new observations are analyzed with the HAZEL code. We first describe the observations (Sects. 2 and 3) and then explain the diagnostic technique (Sect. 4). In Sect. 5 we present the inferred two-dimensional map of the magnetic field vector of the observed prominence, and then we discuss and summarize the results in Sect. 6.

\section{Observations, context data, and prominence morphology}

In this paper, we use observations taken with the TIP-II instrument on 20 May 2011. In particular, we scanned a region of about $40^{\prime \prime}$ wide with the VTT spectrograph, crossing the solar southeast limb where a quiescent prominence was visible in the $\mathrm{H}_{\alpha}$ slit-jaw images. The observation started at 9:44 UT and finished at 11:15 UT. The length of the spectrograph's slit was $80^{\prime \prime}$ with a spatial sampling along the slit of $00^{\prime} 17$ and a scanning step of 0.5 , which provided us with a $80^{\prime \prime} \times 40^{\prime \prime}$ map. Thanks to the adaptive optics system of the VTT we could maintain stable observing conditions (in terms of spatial resolution) during the $95 \mathrm{~min}$ that took the scanning of the prominence. We believe that the spatial resolution of our data lies between $1^{\prime \prime}$ (limited by the scanning step) and 1.'5. During the scanning, the TIP-II instrument recorded the four Stokes parameters around the $1083.0 \mathrm{~nm}$ spectral region. This region contains the chromospheric He I $1083.0 \mathrm{~nm}$ triplet as well as the photospheric Si I $1082.70 \mathrm{~nm}$ line, including an atmospheric water vapor line at $1083.21 \mathrm{~nm}$. The spectral sampling was $1.1 \mathrm{pm}$ and the exposure time per polarization state was $15 \mathrm{~s}$. The TIP-II data 


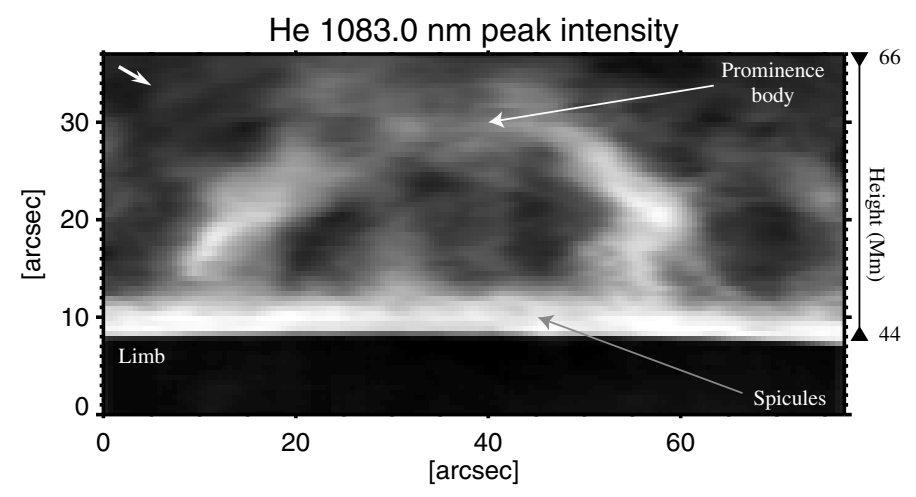

Fig. 1. Peak intensity map of the He I $1083.0 \mathrm{~nm}$ triplet emission profile. The prominence is seen as a bright structure against a dark background. The lower dark part corresponds to the solar limb. The top-left arrow points to solar North. The deprojected height (see Sect. 2) above the solar surface is shown on the right axis. The data was taken 20 May 2011 at 9:44 UT and finished at 11:15 UT on the same day.

reduction process included dark-current, flat-field, and fringes correction, as well as the polarimetric calibration. To improve the signal to noise ratio the data were down-sampled spectrally and spatially along the slit direction, yielding a final spectral and spatial sampling of $4.4 \mathrm{pm}$ and 0.51 , respectively.

The observed prominence can be seen in Fig. 1, were the $X$-axis represents the position along the slit and the $Y$-axis is the scanning direction. The right axis shows the deprojected height over the solar surface. The more vertical appearance of the prominence on both sides of the observed FOV (hereafter, prominence feet), which are connected to each other with a more diffuse horizontal filamentary structure (hereafter, prominence body) shape the prominence as loop-like structure. The feet show more He I peak intensity signal than the prominence body. They may be connecting the prominence body with the chromosphere. In the intensity map, we cannot distinguish finer details within the prominence such as threads, even though the data were obtained during good seeing conditions.

To put the TIP-II observations in context, we made use of data provided by the extreme ultraviolet light telescope (AIA; Lemen et al. 2012) onboard NASA's Solar Dynamics Observatory (SDO; Pesnell et al. 2012), the Solar Terrestrial Relations Observatory (STEREO-B) Extreme UltraViolet Imager (Kaiser et al. 2008), and the Big Bear Solar Observatory (BBSO) high-resolution $\mathrm{H}_{\alpha}$ filter (Denker et al. 1999). Figure 2 displays maps of the prominence as seen in the Fe IX $171 \AA$, Fe XIV $211 \AA$, and He II $304 \AA$ AIA band passes (top panels). The AIA spatial resolution is about $\sim 1$ '. 6 . The white box outlines the TIP-II field-of-view, thus our observations sampled the central part of the prominence as seen in these filters (see Fig. 1). In the $171 \AA$ and $211 \AA$ filters, the prominence is seen as a dark absorption against a bright background. It looks like a sheet made of strands, forming arc structures. In the $304 \AA$ filter the prominence appearance is rather different. This filter shows radiation coming from plasma at $\log T=4.7$. In this case, it can be seen as a large and dark envelope with a horn-like (U-shaped) structure in the top (see arrows in Fig. 2, top right panel). These "horns" may be indicating the presence of a coronal cavity right above the prominence (Régnier et al. 2011).

In the $\mathrm{H}_{\alpha}$ image (bottom left panel), the prominence body is seen in emission outside the solar disk while the prominence feet are seen in absorption, hence darker that the surroundings, because they lie within the solar disk. The shape resembles the prominence as seen in the peak intensity of the He I $1083.0 \mathrm{~nm}$ triplet (Fig. 1). The last two panels of Fig. 2 display STEREO Fe XII $195 \AA$ and He II $304 \AA$ observations. The prominence can be clearly seen in absorption (i.e., as a filament), which allows us to know its position on the solar disk and the angle $\theta$ between our LOS and the solar radius vector through the observed point (hereafter the local solar vertical). This light scattering angle is necessary to properly invert the Stokes profiles (see Sect. 4). In this case, $\theta \approx 70^{\circ}$, on average. The exact values of the $\theta$ angle at each pixel of the TIP-II slit is taken into account in the analysis of the profiles with HAZEL. We can also calculate the real height $h$ over the solar surface from the apparent height $h^{\prime}$ as (see Fig. 3 from Merenda et al. 2006):

$h=\frac{R_{\odot}+h^{\prime}}{\cos \left(90^{\circ}-\theta\right)}-R_{\odot}$.

In Fig. 3 we sketch the geometry of the prominence. In particular, we show a top view of the prominence using STEREO-B contour lines (right side). The filament has a length of about $138^{\prime \prime}(\sim 100 \mathrm{Mm})$ and a width of $15^{\prime \prime}$. The angle between the LOS and the long axis of the prominence is $\alpha=90^{\circ}+\beta$, where $\beta \sim 17^{\circ}$ is the angle that the prominence forms with the meridian, measured counterclockwise. In the right hand side of Fig. 3, we define the reference system with the $Y$ and $Z$-axis contained in the sky-plane. Finally, SDO/HMI magnetograms provided us with a map of the photospheric magnetic flux. They show that in the right hand side (solar west) of the prominence, positive polarity flux dominates the photosphere. This information will help us determine the chirality of the filament, once we have inferred the magnetic field vector in the prominence body.

We classify the observed prominence as of quiescent type, meaning that it is located outside active regions. Quiescent prominences are often characterized as sheets of plasma standing vertically above the PIL and showing prominence threads. When these threads are vertically oriented, quiescent prominences are often classified as the hedgerow type. The attained spatial resolution in TIP-II observations prevented us from resolving any prominence small-scale structures. However, we do see strands in the EUV images. At high latitudes, these show motions that are mainly parallel to the solar limb, similar to those described by Chae et al. (2008). At low latitudes, the motions seem to be perpendicular to the limb and are typical of interactive hedgerow prominences (Pettit 1943; Hirayama 1985). The long-term evolution of the prominence can be seen in an SDO/AIA Fe XIV $211 \AA$ movie available in the online edition along with Fig. 2 . In the movie, the evolution of the finescale structures can be very well appreciated. Interestingly, at 16:30 UT ( $5 \mathrm{~h}$ after the TIP-II observation) and for no apparent reason, the prominence begins to rise and erupts (not shown in the movie). This prominence may be similar to the one observed by Athay et al. (1983), which also erupted shortly after the observations. The eruption is slow and lasted $15 \mathrm{~h}$ until 21 May at 7:00 UT. Remarkably, we have detected apparent spiraling motions in the feet of this prominence on 19 May 2011 using sit-and-stare slit observations and TIP-II (Orozco Suárez et al. 2012).

\section{Analysis of the polarization signals}

Individual He I $1083.0 \mathrm{~nm}$ triplet emission profiles recorded in this quiescent prominence are shown in Fig. 4. The Stokes $I$ profiles are normalized to their maximum peak value (first column). They show the fine structure of the He I $1083.0 \mathrm{~nm}$ 

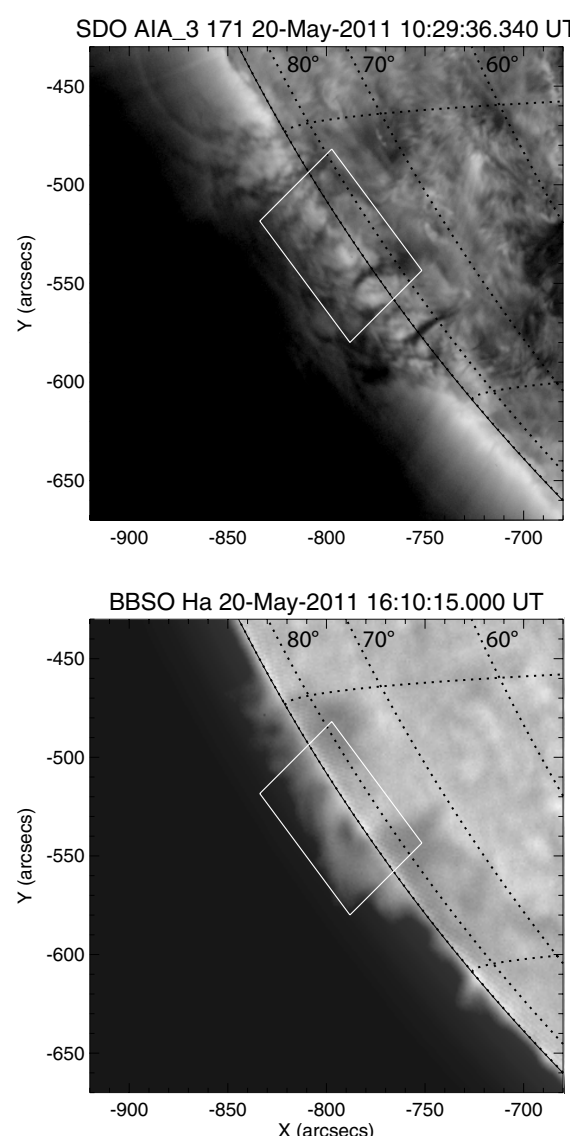

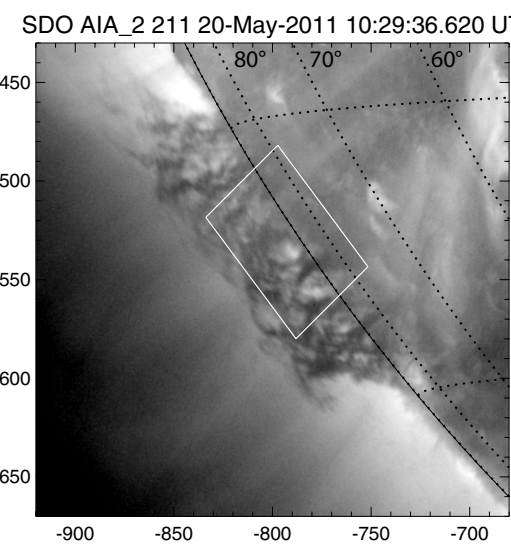

STEREO_B SECCHI EUVI 195 20-May-2011 10:00:52.868 UT

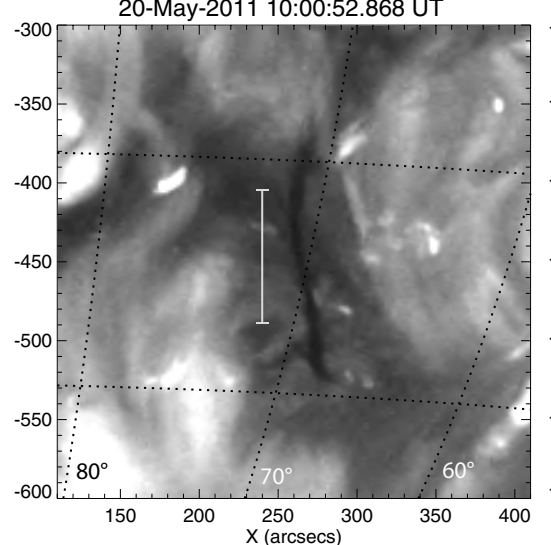

SDO AIA_4 304 20-May-2011 10:29:44.140 UT

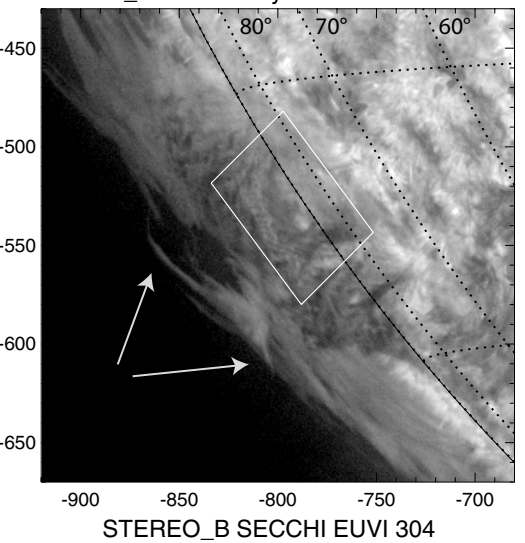

STEREO_B SECCHI EUVI 304

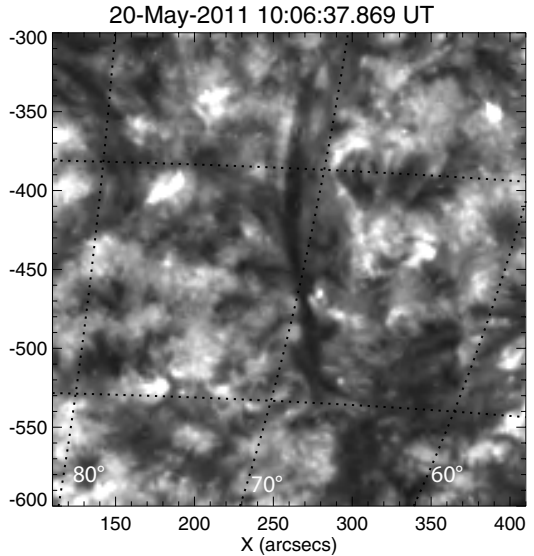

Fig. 2. Illustrations of the observed prominence as seen in SDO/AIA, STEREO/EUVI, and the BBSO. Top panels correspond to Fe IX $171 \AA$, Fe XIV $211 \AA$, and He II $304 \AA$ AIA band-pass filter images. Bottom panels are BBSO H $\alpha$ broad-band image, and Fe XII $195 \AA$ and He II $304 \AA$ STEREO-B/EUVI band-pass images. All images were taken on 20 May 2011, the same day the TIP-II observations were carried out. The white box represents the TIP-II field-of-view. Lines of constant Stonyhurst heliographic longitude and latitude on the solar disk are overplotted. Axis are in heliocentric coordinates. The arrows pinpoint the location of horn-like structures in He II $304 \AA$. The TIP-II slit virtual position can be seen in the bottom central panel. The quiescent prominence can be clearly seen in the AIA images, as well as in $\mathrm{H}_{\alpha}$. In STEREO-B, it can be seen as a dark, elongated structure. The temporal evolution of the prominence in the SDO/AIA Fe XIV $211 \AA$ channel is available in the online edition.

triplet, i.e., a weak blue component at $1082.9 \mathrm{~nm}\left({ }^{3} \mathrm{~S}_{1}-{ }^{3} \mathrm{P}_{0}\right)$ separated about $0.12 \mathrm{~nm}$ from the two blended components located at about $1083.03 \mathrm{~nm}\left({ }^{3} \mathrm{~S}_{1}-{ }^{3} \mathrm{P}_{1}\right.$ and $\left.{ }^{3} \mathrm{~S}_{1}-{ }^{3} \mathrm{P}_{2}\right)$ that produce a stronger emission peak. The next columns represent the Stokes $Q / I_{\max }, U / I_{\max }$, and $V / I_{\max }$ signals, normalized to each of their Stokes $I$ maximum value ${ }^{2}$ (hereafter for simplicity $Q / I$, $U / I$, and $V / I)$. The Stokes $Q / I$ and $U / I$ polarization signals are given after rotating the reference system so that the positive reference direction for Stokes $Q$ is the parallel to the nearest limb. They show nonzero profiles with a prototypical Stokes $I$ shape but with the blue component absent. This is the signature of scattering polarization, whose true physical origin is atomic level polarization.

The mere presence of the Stokes $U / I$ signal suggests the presence of a magnetic field inclined with respect to the local vertical direction, according to the Hanle effect theory. Both Stokes $Q / I$ and $U / I$ show polarization only in the red component of the triplet, as expected for the case of a prominence observed against the dark background of the sky (Trujillo Bueno et al. 2002; Trujillo Bueno \& Asensio Ramos 2007). The blue component does not show any linear polarization signal in weakly magnetized optically thin plasmas observed against the dark

\footnotetext{
2 In off-limb observations, it is typical to normalize the polarization signals to their Stokes $I$ peak value since there is no emission in the continuum.
}

background of the sky, because its linear polarization can only be due to the selective absorption of polarization components caused by the atomic polarization in the (metastable) lower level of the He I $1083.0 \mathrm{~nm}$ triplet. Since the upper level of the blue component $\left({ }^{3} \mathrm{P}_{0}\right)$ has $J=0$, it cannot be polarized, so that the emitted radiation has to be unpolarized. Only when dichroic effects become important (in plasmas with a sizable optical depth or when observing plasma structures (e.g., quiescent filaments) against the bright background of the solar disk), the blue component may show polarization. According to our observations, the prominence material we observed had to present a very small optical thickness (see Fig. 9), so that the slab model is suitable for interpreting of the emission profiles.

Finally, the Stokes V/I signals show the typical circular polarization profiles dominated by the Zeeman effect, i.e., two lobes of opposite sign with a zero-crossing point. Overall, Fig. 4 nicely illustrates the joint action of the Hanle and Zeeman effect in the He I $1083.0 \mathrm{~nm}$ triplet. We display three different cases: (a) represents a pixel in which the circular polarization signal is well above the noise level and shows the prototypical antisymmetric Stokes $V$ profiles dominated by the longitudinal Zeeman effect; (b) corresponds to a pixel with negligible circular polarization; and (c) shows a profile with net (wavelength-integrated) circular polarization in Stokes $V / I$. The physical origin of the net circular polarization in case (c) may be due to the atomic orientation in the energy levels and/or to correlated magnetic field and 
D. Orozco Suárez et al.: The magnetic field vector configuration of a solar prominence

TOP VIEW

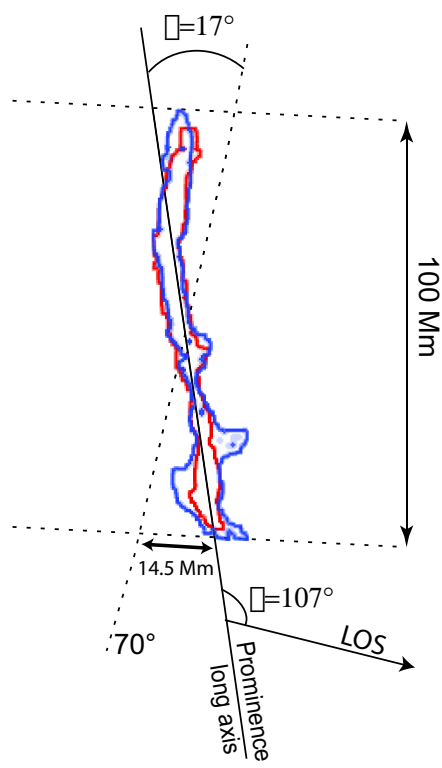

SIDE VIEW

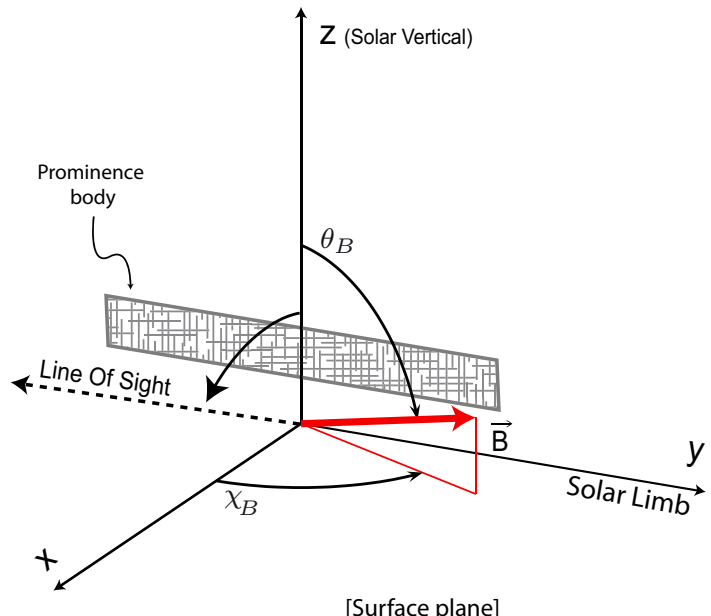

Fig. 3. Left: sketch of the prominence as seen on disk (top view). The blue and red contours outline the filament as seen in STEREO-B/EUVI Fe XII $195 \AA$ and He II $304 \AA$ band-pass images (see Fig. 2). The arrow points to the line-of-sight (LOS) direction. The dotted lines correspond to constant Stonyhurst heliographic longitude and latitude positions on the solar disk. The prominence sits in the $70^{\circ}$ longitude, which gives a scattering angle of $\theta=70^{\circ}$. Right: geometry of the problem. The inclination of the magnetic field $\theta_{B}$ is measured from the $z$-axis (solar vertical) and the azimuth $\chi_{B}$ counterclockwise from the $x$-axis, contained on the surface plane. The graph shows the angle $\theta$ between the LOS direction and the local vertical, which corresponds to the light scattering angle.

velocity gradients along the LOS (see Martínez González et al. 2012, and more references therein).

Figure 5 displays Stokes $Q, U$, and $|V|$ wavelength integrated maps. The Stokes $Q$ map closely resembles the Stokes $I$ peak intensity map displayed in Fig. 1. Here, the feet and the prominence body are clearly distinguishable from the background. In the case of Stokes $U$, the map shows clear signals in the prominence feet but not in the prominence body. Interestingly, the sign of Stokes $U$ changes from positive (white) in the left feet to negative (black) in the right feet. There are some positive signals in the right feet as well. Thanks to the long exposure times per slit position, we could also detect clear circular polarization signals along the prominence. In this case, the strongest Stokes $V$ signals are concentrated in the feet of the prominence, while in the body they are rather weak and contaminated by the noise. In the bottom right hand panel we display contours delimiting areas where Stokes $V$ surpasses three and five times their intrinsic noise levels $^{3} \sigma$. Here, that the Stokes $V$ signals dominate in the feet is evident. As we explain in Sect. 4.2, the mere detection of circular polarization is important for distinguishing between the different magnetic field vector orientations compatible with the observed linear polarizations signals. Moreover, it helps determining the strength of the magnetic field when the He I $1083.0 \mathrm{~nm}$ triplet is in the saturation regime $(B \gtrsim 10 \mathrm{G})$.

\section{Interpretation of the polarization signals}

\subsection{Diagnostics of the $\mathrm{Hel} 1083.0 \mathrm{~nm}$ triplet}

The He I $1083.0 \mathrm{~nm}$ triplet is suitable for determining the magnetic field vector in chromospheric and coronal structures. The

\footnotetext{
3 Since the Stokes profiles are normalized to the peak amplitude of Stokes $I$, the noise level $\sigma$ (or the signal-to-noise ratio) differs from pixel to pixel, depending on the amplitude of the detected signal.
}

main reason is that there are three physical processes able to generate and/or modify circular and linear polarization signals in the He I $1083.0 \mathrm{~nm}$ triplet: the Zeeman effect, atomic polarization resulting from anisotropic radiation pumping, and the Hanle effect. All these effects can be studied and understood within the framework of the quantum theory of spectral line polarization (Landi Degl'Innocenti \& Landolfi 2004; Trujillo Bueno et al. 2002; Trujillo Bueno \& Asensio Ramos 2007), and they provide a broad sensitivity to the magnetic field vector from very weak fields to hecto- and kilo-Gauss fields. To interpret the observations, we use the HAZEL code. This code is able to infer the magnetic field vector from the emergent Stokes profiles of the He I $1083.0 \mathrm{~nm}$ triplet considering the joint action of atomic level polarization and the Hanle and Zeeman effects. The following assumptions made by the HAZEL code are particularly fulfilled in solar prominences:

- We chose a simple radiative transfer scenario where the radiation is produced by a slab of constant physical properties. When neglecting or including the magneto-optical effect terms of the propagation matrix, the solution to the radiative transfer equation has an analytic expression (Asensio Ramos et al. 2008; Trujillo Bueno et al. 2005). Under this approximation, the optical thickness of the slab, $\Delta \tau$, is a free parameter that can be inferred from the Stokes $I$ profile at each point of the observed field of view.

- The slab atoms are illuminated from below by the (fixed and angle-dependent) photospheric solar continuum radiation tabulated by Cox (2000), producing population imbalances and quantum coherence in the levels of the He I atoms. This produces polarization in the emitted radiation.

- The atomic level polarization are calculated assuming complete frequency redistribution, which is a reliable approximation for modeling the observed polarization signatures in the He I $1083.0 \mathrm{~nm}$ triplet (Trujillo Bueno \& Manso Sainz 2002; Trujillo Bueno 2010). 

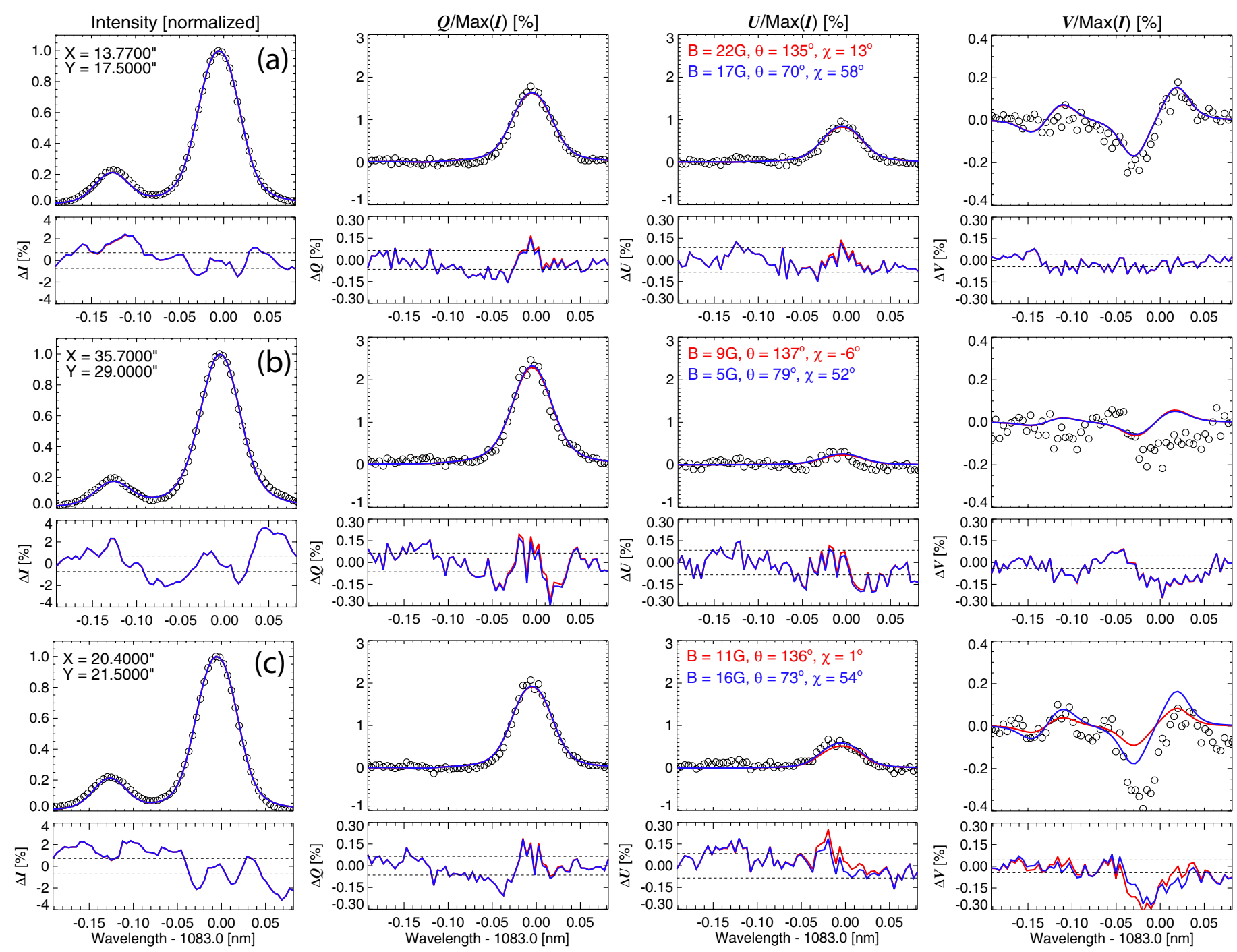

Fig. 4. Observed and best-fit He1 $1083.0 \mathrm{~nm}$ triplet Stokes profiles corresponding to different pixels in the prominence. Stokes $I$ is normalized to unity, while Stokes $Q, U$, and $V$ are normalized to their Stokes $I$ maximum peak value. Open dots represent the observations and solid lines stand for the theoretical profiles obtained by HAZEL. Blue and red represent two different solutions. In this case, we plot the quasi-horizontal solution (blue) and the corresponding one to the $90^{\circ}$ ambiguity (red), which corresponds to the quasi-vertical solution (see Sect. 4). The bottom subpanels display the difference between the observed and the synthetic profiles. The horizontal dashed lines in the subpanels stand for the mean standard deviation of the noise: $\bar{\sigma}_{I, Q, U, V}= \pm[0.72,0.065,0.085,0.045] \%$. They were calculated by averaging the standard deviation at a single wavelength point in the continuum and over all pixels showing polarization signal amplitudes above three times their corresponding noise level, in Stokes $Q$, $U$, or $V$. The legend in the Stokes $I$ panels gives the location of the pixel in arcseconds and the one in Stokes $U / I$ gives the values of the field strength, inclination, and azimuth retrieved for each pixel and for the two $90^{\circ}$ ambiguous solutions. The positive reference direction for Stokes $Q$ is the parallel to the solar limb. Panels a), b), and c) represent a prototypical He1 $1083.0 \mathrm{~nm}$ triplet profile with significant linear and circular polarization signals, a profile with noisy Stokes $V$ signal, and one whose Stokes $V$ signal shows an anomalous profile shape.

- We work in the collisionless regime, in which the atomic polarization is controlled by radiative processes. No reliable estimations of the depolarizing collisional rates are available for the $\mathrm{He}$ I atom.

The inference strategy in HAZEL consists in comparing the observed Stokes profiles with synthetic guesses of the signals. Within HAZEL, the slab model is fully described using seven free parameters: the optical slab's thickness $\Delta \tau$ at the central wavelength of the red blended component, the line damping parameter $a$, the thermal velocity $\Delta v_{\mathrm{D}}$, the bulk velocity of the plasma $v_{\mathrm{LOS}}$, and the strength $B$, inclination $\theta_{B}$, and azimuth $\chi_{B}$ of the magnetic field vector with respect to the solar vertical. To fully characterize the incoming radiation, we also need to determine the height $h$ above the limb of each prominence point and the $\theta$ angle, i.e., the angle that forms the LOS direction with the local vertical (see Fig. 3). These two parameters fix the degree of anisotropy of the incident radiation field and are kept constant since we can determine them directly from the observations. It is important to properly determine the real height above the limb, as well as the $\theta$ angle, to avoid imprecisions in the determination of the field vector orientation. In our case, these two parameters were determined using STEREO data. Finally, note that only pixels whose linear polarization signals exceeded five times the noise level are inverted in order to minimize the effect of noise on the inferences.

\subsection{Ambiguities}

The behavior of the He I $1083.0 \mathrm{~nm}$ triplet polarization signals depends in a very complicated manner on the orientation of the 

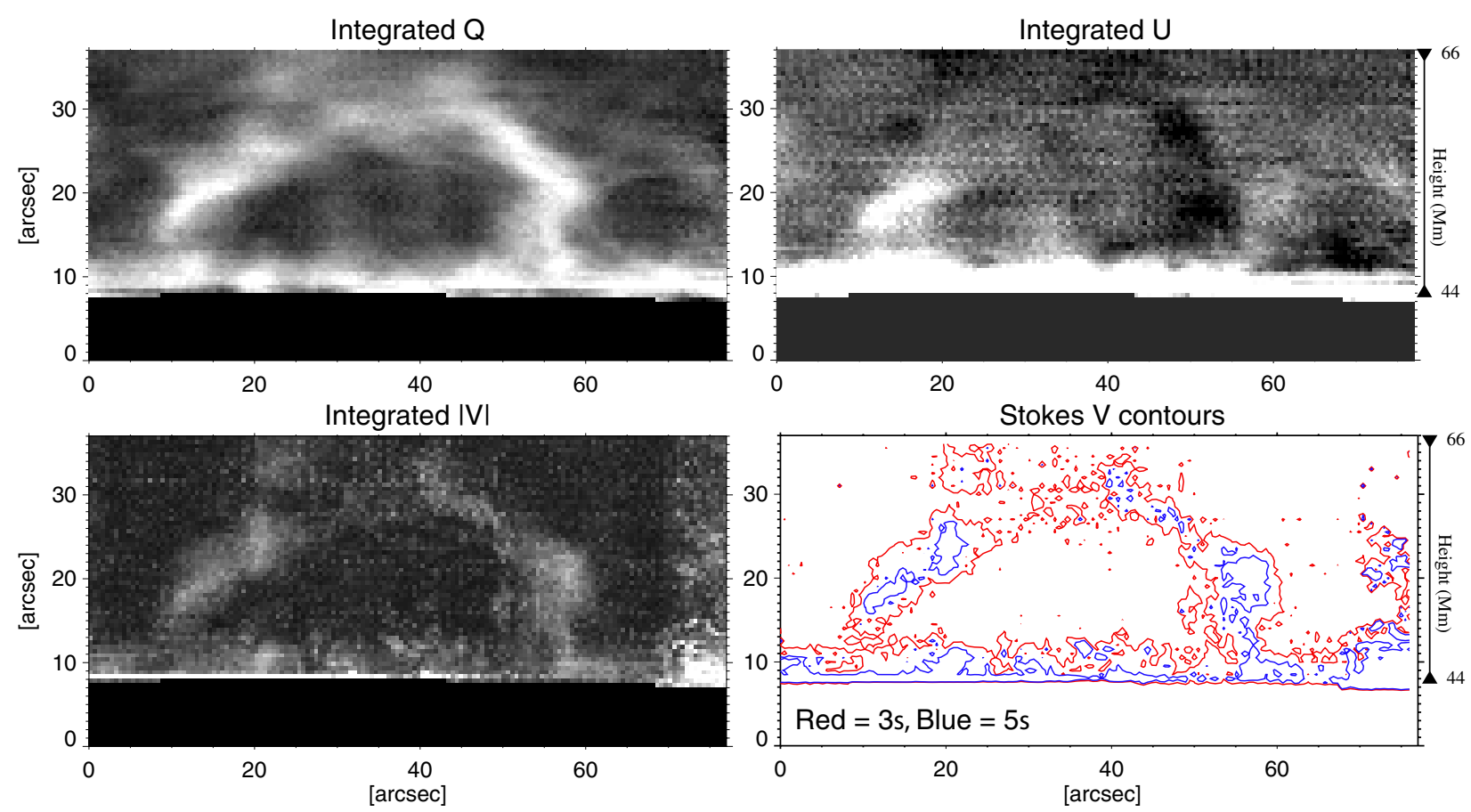

Fig. 5. Stokes $Q, U$, and $|V|$ wavelength integrated maps calculated by integrating the observed Stokes profiles. The integral covered 21 wavelength samples centered on the position of maximum emission. The bottom right panel shows contour plots representing the areas where the peak amplitude of the Stokes $|V|$ signal surpasses three and five times the noise level $\sigma$. Note that $\sigma$ is pixel dependent since each Stokes parameter is normalized to its Stokes I maximum amplitude. As in Fig. 1, the bottom part represents the limb and the right axis the height in Mm.
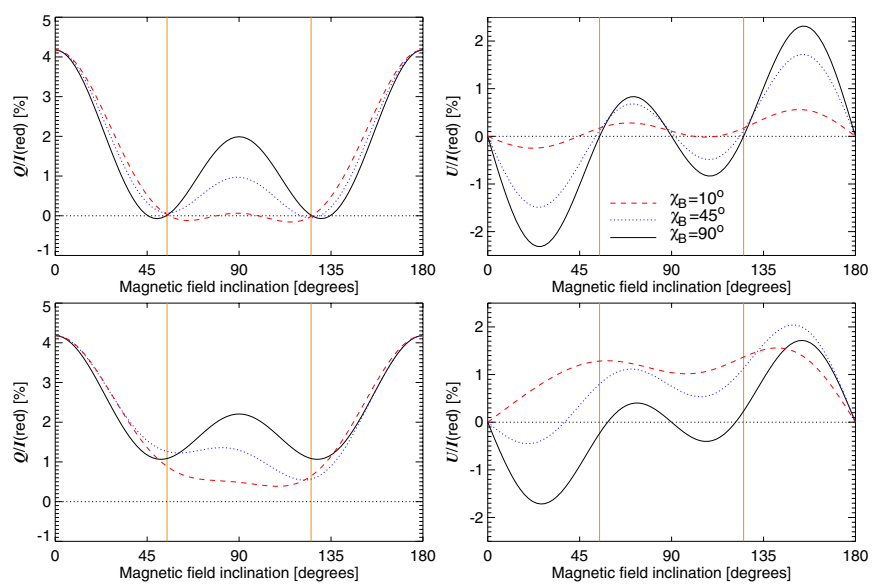

Fig. 6. Variation in Stokes $Q / I$ and $U / I$ amplitudes of the He I $1083.0 \mathrm{~nm}$ triplet red component with the magnetic field inclination for three different magnetic field azimuth values, in the $90^{\circ}$ scattering geometry. The top panels correspond to the saturation regime, $B>10 \mathrm{G}$, and the bottom panels to no saturation, $B=1 \mathrm{G}$. The rest of parameters for synthesizing the Stokes profiles were: $\Delta \tau=0.6$ at the central wavelength of the red blended component, $a=0.5, \Delta \lambda_{\mathrm{D}}=7 \mathrm{~km} \mathrm{~s}^{-1}$, and $h=10^{\prime \prime}$. The vertical lines are the Van Vleck angles at $54.74^{\circ}$ and $125.26^{\circ}$. Here, the positive reference direction for Stokes $Q$ corresponds to $\chi_{B}=90^{\circ}$.

magnetic field with respect to the LOS and on the inclination of the magnetic field with respect to the local solar vertical. This dependence can be clearly seen in the Hanle saturation regime. In this case, the amplitude of the linear polarization signals is given by Eq. (6) of Trujillo Bueno (2010)

$\frac{Q}{I} \approx-\frac{3}{4 \sqrt{2}} \sin ^{2} \Phi_{B}\left(3 \cos ^{2} \theta_{B}-1\right) \mathcal{F}$ where $\Phi_{B}$ is the angle between the magnetic field vector and the LOS, $\theta_{B}$ is the inclination of the magnetic field vector with respect to the local solar vertical, and we take the Stokes $Q$ reference direction the parallel to the projection of the magnetic field onto the plane perpendicular to the LOS (i.e., the reference direction for which Stokes $U / I=0$ ). The quantity $\mathcal{F}$ is determined by solving, for the unmagnetized case, the statistical equilibrium equations for the elements of the atomic density matrix. The $\left(3 \cos ^{2} \theta_{B}-1\right)$ term tells us that Stokes $Q / I$ is identically zero when $\theta_{B}$ is $54.74^{\circ}$ and $125.26^{\circ}$. These are the so-called Van Vleck angles. Given the non linear function of Eq. (2), we can expect that Stokes $Q / I$ takes the same value for different field inclinations. This non-linear behavior gives rise to the so-called $90^{\circ}$ ambiguity of the Hanle effect. The $90^{\circ}$ ambiguity is associated with the Van Vleck angles because the magnetic field inclination of two $90^{\circ}$ ambiguous solutions lie on both sides of any of the Van Vleck angles. There can be particular orientations of the field vector where this ambiguity does not take place (e.g., Merenda et al. 2006). Additional material about how this ambiguity works and how to deal with it can be found in Asensio Ramos et al. (2008), Casini et al. (2005, 2009), and Merenda et al. (2006).

A simplified illustration of why the $90^{\circ}$ ambiguity appears can be found in Fig. 6. The figure shows the dependence of the Stokes $Q / I$ and $U / I$ amplitudes, measured at the central wavelength of the red blended component of the He I $1083.0 \mathrm{~nm}$ triplet, with the magnetic field inclination for different azimuth values. All calculations were done with the HAZEL code. The graph is similar to that of Fig. 9 in Asensio Ramos et al. (2008) but for the $90^{\circ}$ scattering geometry. The top panels correspond to a magnetic field strength $B=11 \mathrm{G}$, for which the He I $1083.0 \mathrm{~nm}$ triplet is practically in the saturation regime of the upper-level Hanle effect. The bottom panels is for $B=1 \mathrm{G}$. We note the non linear dependence of the Stokes $Q / I$ and Stokes $U / I$ 


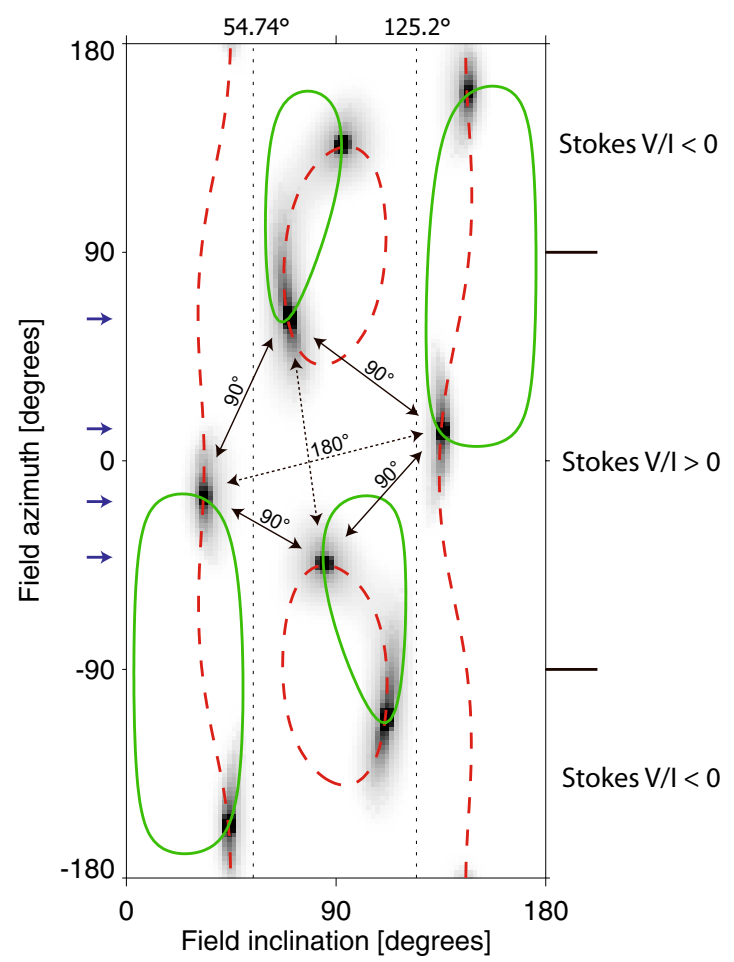

Fig. 7. Variation of the merit function with the inclination $\theta_{B}$ and azimuth $\chi_{B}$ of the field vector. The minima are located at eight different positions. Four of them correspond to a field vector pointing to the observer, i.e., positive Stokes $V$ signals. These four solutions are connected through the $90^{\circ}$ ambiguity and the $180^{\circ}$ ambiguity of the Hanle effect (see arrows). The vertical dotted lines correspond to the Van Vleck angles $54.74^{\circ}$ and $125.26^{\circ}$. The merit function here has been calculated using the parameters derived from the inversion of profile (a) in Fig. 4: $B=17 \mathrm{G}, \Delta \tau=1.13, \theta=71.4^{\circ}, h=61^{\prime \prime} 8, \Delta \lambda_{\mathrm{D}}=6 \mathrm{~km} \mathrm{~s}^{-1}$, and $a=0.33$. The contours delimit connected regions by the same value of Stokes $Q / I$ (dashed) and $U / I$ (solid).

amplitude signals with the inclination angle. Because of this non-linear dependence, there may be particular magnetic field vector orientations giving rise to the same Stokes $Q / I$ or $U / I$ amplitude signals. For instance, a magnetic field configuration having $90^{\circ}<\theta_{B}<125.26^{\circ}$ and $\theta_{B}<54.74^{\circ}$ can potentially give rise to the same Stokes $Q / I$ and $U / I$ amplitudes by appropriately modifying the field azimuth.

In the Hanle saturation regime, the linear polarization signals depend only on the inclination of the magnetic field $\Phi_{B}$ with respect to the LOS and on the inclination of the magnetic field $\theta_{B}$ with respect to the local solar vertical (see Eq. (1)). In the unsaturated case, the variation in Stokes $Q / I$ and $U / I$ amplitudes with $\theta_{B}$ also depends on the field strength $B$. As a result, the Stokes $Q / I$ signals may be always positive for any field inclination and azimuth. Stokes $U / I$ can also be always positive when $\chi_{B}=0^{\circ}$. Moreover, the Stokes $Q / I$ and $U / I$ amplitude signals are not symmetrical around $\theta_{B}=90^{\circ}$ in the unsaturated regime. This strong dependence on field strength helps determine not only the orientation of the magnetic field vector from the analysis of the linear polarization signals, but also the field strength itself.

Besides the $90^{\circ}$ ambiguity, there is another ambiguity called the $180^{\circ}$ ambiguity of the Hanle effect, similar to the well-known azimuth ambiguity of the Zeeman effect. In this case, the linear polarization signals do not change when we rotate the field vector $180^{\circ}$ around the LOS axis (a rotation of the magnetic field in the plane perpendicular to the LOS). In the case of $90^{\circ}$ scattering geometry, profiles differing by $180^{\circ}$ in the inclination
$\theta_{B}^{*}=180^{\circ}-\theta_{B}$ (in the plane perpendicular to the LOS) give rise to the same linear polarization profiles but with an azimuth $\chi_{B}^{*}=-\chi_{B}$.

In summary, determination of the orientation of the field vector from the linear polarization profiles of the He1 $1083.0 \mathrm{~nm}$ triplet is affected by two ambiguities: the $180^{\circ}$ azimuth ambiguity and the $90^{\circ}$ ambiguity. There is another pseudo-ambiguity associated with Stokes $V / I$. When the signal-to-noise-ratio is not high enough to detect the circular polarization signal, we cannot determine if the field vector is pointing to the observer or away from the observer. In $90^{\circ}$ scattering geometry, it would correspond to an ambiguity in the azimuth as $\chi_{B}^{\prime}=180^{\circ}-\chi_{B}$ that corresponds to specular reflections in the plane perpendicular to the LOS. Thus, in total we can potentially have eight possible solutions compatible with a single observation.

As in Fig. 13 of Asensio Ramos et al. (2008), we show in Fig. 7 an example of how the merit function, calculated as $\chi^{2}=\sum_{i=1}^{2}\left[S_{i}^{\text {syn }}\left(\lambda_{\text {red }}\right)-S_{i}^{\text {obs }}\left(\lambda_{\text {red }}\right)\right]^{2}$, varies with the inclination $\theta_{B}$ and azimuth $\chi_{B}$ of the field vector. In the previous expression, $i$ represents Stokes $Q / I$ and $U / I$ and $\lambda_{\text {red }}$ is the wavelength position of the He1 $1083.0 \mathrm{~nm}$ red component. The figure shows eight local minima (dark places). Four of them are incompatible with the observations because they require Stokes $V / I$ profiles with a negative sign ${ }^{4}$ (field vector pointing away from the LOS), and we observe a Stokes $V / I$ with a positive sign. The four other solutions (marked with arrows in the left axis) are connected through the $90^{\circ}$ ambiguity and the $180^{\circ}$ ambiguity as the solid and dotted arrow lines show. The contour lines represent isolines along which the merit function has constant Stokes $Q / I$ and $U / I$ values. They cross where the merit function has a local minima.

In practice, to determine all possible solutions we proceeded as follows. First, we checked the sign of Stokes $V / I$ to delimit the range of possible azimuths. In our prominence, Stokes $V / I$ always had a positive sign, thus $-90^{\circ}<\chi_{B}<90^{\circ}$, which means that the LOS magnetic field vector component is always pointing toward the observer. There are four potential solutions within the parameter range $-90^{\circ}<\chi_{B}<90^{\circ}$ and $0^{\circ}<\theta_{B}<180^{\circ}$. Therefore, to explore all possible solutions, we performed six different inversions: three inversion runs that allows the azimuth $\chi_{B}$ to vary between $-90^{\circ}$ and $0^{\circ}$ and the inclination to vary within the ranges $0^{\circ}<\theta_{B}<54.74^{\circ}, 54.74^{\circ}<\theta_{B}<125.26^{\circ}$, and $125.26^{\circ}<\theta_{B}<180^{\circ}$, and three other runs for $0^{\circ}<\chi_{B}<90^{\circ}$ and the same inclination ranges. Only four of these inversions will provide a magnetic field vector configuration whose emergent Stokes profiles are compatible with the observations. This approach is rather rough, although it allows the complete parameter range to be explored. The present version of the HAZEL code does this work automatically, using the analytical expressions in the saturation regime as a basis. Also, HAZEL provides a discrete solution for each pixel. The above strategy allows us to impose a continuity condition, since the solutions will not jump from one possible solution to another for each of the inversion runs.

\section{Inversion results}

Figure 4 shows observed and best-fit Stokes profiles of the He I $1083.0 \mathrm{~nm}$ triplet corresponding to three different locations on the prominence. The subpanels show the difference between the best-fit and the observed profiles. Case (a) shows a profile where the linear and circular polarization signals are prominent

\footnotetext{
4 Here, the sign of Stokes $V$ is defined as the sign of the Stokes $V / I$ blue lobe amplitude.
} 

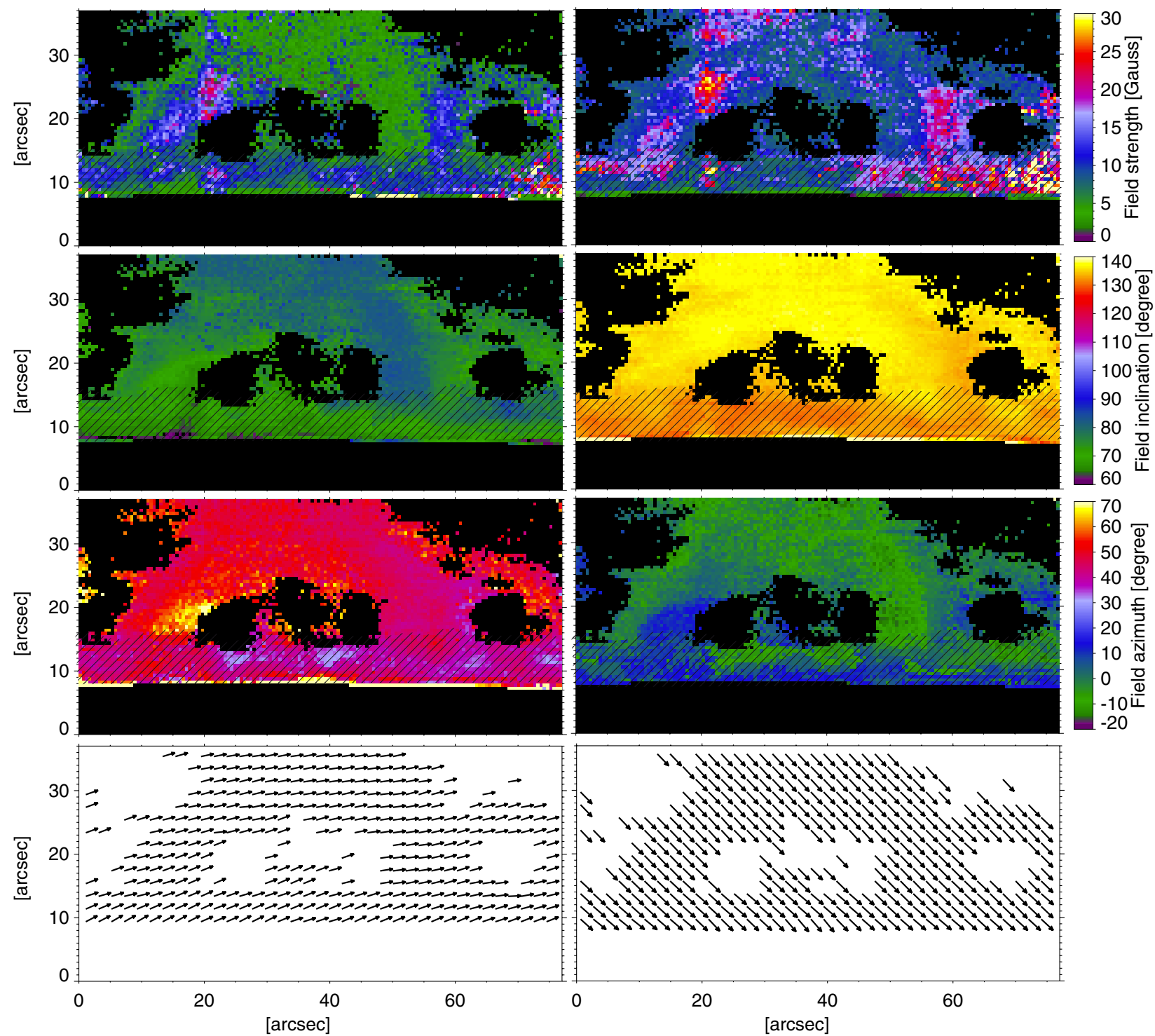

Fig. 8. Field strength, inclination, and azimuth maps resulting from the inversion of the observed Stokes profiles with the HAZEL code. Each column represents one of the two $90^{\circ}$ ambiguous solutions, quasi-horizontal (left) and quasi-vertical (right). As in Fig. 1, the black bottom region represents the solar disk. The rest of the dark areas correspond to pixels whose Stokes $Q / I$ and $U / I$ signals did not exceed 5 times their corresponding noise levels.

above the noise level. The fits are good in Stokes $Q / I, U / I$, and $V / I$, and the residuals are very small. Profiles in case (b) correspond to a point where the Stokes $U / I$ and $V / I$ signals are barely above the noise level. The fit in Stokes $Q / I$ is good. Case (c) represents an infrequent pixel where the Stokes $V / I$ signal shows net circular polarization. Again, the fits are good except for Stokes $V / I$. The version of HAZEL we applied neglects atomic orientation and possible correlations between the magnetic field and the velocity gradients along the $\operatorname{LOS}^{5}$. Cases (a) and (c) are in the Hanle saturation regime, $B>10 \mathrm{G}$. In the same panels we show both the quasi-vertical and quasi-horizontal solutions, i.e., one solution and its corresponding $90^{\circ}$ ambiguous solution. We pay attention to the difference between the two fits: it is negligible and at the noise level which means that the Stokes $Q / I$, $U / I$, and $V / I$ profiles are indistinguishable for two different magnetic field orientations. Two compatible solutions are found not

\footnotetext{
5 Given that the fractional number of pixels showing anomalous Stokes $V$ profiles is very small, we do not find it justified to increase the complexity of our model assumptions.
}

Table 1. Summary of results.

\begin{tabular}{lcccc}
\hline \hline Quasi- & $B[\mathrm{G}]$ & $\theta_{B}\left[{ }^{\circ}\right]$ & $\chi_{B}\left[{ }^{\circ}\right]$ & $\chi^{\dagger}\left[{ }^{\circ}\right]$ \\
\hline -horizontal (Dextral) & 6.9 & 77.0 & 49.0 & 58 \\
-vertical (Sinistral) & 11.7 & 136.0 & -1.5 & 108 \\
-horizontal (Sinistral - $180^{\circ}$ ) & 6.4 & 77.0 & -49.0 & 156 \\
-vertical (Sinistral $-180^{\circ}$ ) & 7.8 & 33.8 & 1.2 & 106 \\
\hline
\end{tabular}

only for two different magnetic field orientations but also for two different field strengths as seen in the Stokes $U / I$ legend. Only in case (c) do the Stokes $V / I$ fits differ, both of them fitting the profile within the noise level of the data.

The field strength, inclination, and azimuth maps of the prominence obtained from the analysis of the Stokes profiles with HAZEL and corresponding to two compatible solutions via the $90^{\circ}$ ambiguity are displayed in Fig. 8. The left hand panels correspond to a solution where the inclination of the field vector is almost perpendicular to the local vertical (quasi-horizontal 
solution) and the right hand panels to a solution where the field vector is more vertical than horizontal (quasi-vertical solution). The analysis depicted an average field strength of about $7 \mathrm{G}$ and $12 \mathrm{G}$ for the quasi-horizontal and quasi-vertical solutions, respectively. These values are around the Hanle saturation regime, which emphasizes the importance of detecting Stokes $V$ in order to better constrain the strength of the inferred magnetic field. The maps show that the field strength varies smoothly along the prominence. Only in the left hand part (feet) of the prominence is there a region where the field is stronger than the average, with field strength values up to 25 Gauss for the quasi-horizontal case and 30 Gauss for the quasi-vertical one. A slight increase in the field strength is also noticeable in the right feet. Since for fields stronger than $10 \mathrm{G}$ it is only possible to determine the orientation of the field vector from the Stokes $Q / I$ and $U / I$ profiles, it is important to detect Stokes $V / I$ to help determine the field strength. In this case, the effective exposure time was high enough to measure significant Stokes $Q / I, U / I$, and $V / I$ signals above the noise level (see Fig. 5).

In the quasi-horizontal solution, the inclination of the magnetic field vector, measured from the local vertical, varies from about $70^{\circ}$ at the left part of the prominence to $\sim 90^{\circ}$ on the right hand side. The field inclination is about $135^{\circ}$ for the quasivertical solution. Regarding the magnetic field azimuth, which is measured counterclockwise from the LOS direction, it is about $49^{\circ}$ for the quasi-horizontal case, and it varies between $10^{\circ}$ and $-10^{\circ}$ for the quasi-vertical case. We can estimate the angle between the prominence long axis and the magnetic field vector as $\chi^{\dagger}=\alpha-\chi_{B}$ measured clockwise from the PIL, with $\alpha=107^{\circ}$ the angle between the LOS and the PIL. In this case $\chi^{\dagger}$ is about $58^{\circ}$ for the quasi-horizontal solution and $108^{\circ}$ for the quasi-vertical solution. The latter implies that the field vector is almost perpendicular to the filament PIL, which we may take as an extra reason to go for the quasi-horizontal solution.

The magnetic field vector configurations we show in Fig. 8 correspond to two possible solutions, related by the $90^{\circ}$ ambiguity. All compatible solutions, including those resulting from the $180^{\circ}$ ambiguity of the Hanle effect, are listed in Table 1. The table gives the average values of the inferred magnetic field strength $B$, field inclination $\theta_{B}$, field azimuth $\chi_{B}$, and the angle between the magnetic field vector and the PIL $\chi^{\dagger}$. The table also specifies the chirality of the prominence, i.e., whether the solution is of dextral or sinistral chirality. The chirality can be determined using the information about the sign of the photospheric magnetic field, which is positive (negative) on the right (left) hand side of the prominence. Dextral chirality corresponds to angles between $0^{\circ}<\chi^{\dagger}<90^{\circ}$ and sinistral chirality to angles between $90^{\circ}<\chi^{\dagger}<180^{\circ}$ (Martin 1998). Since $\chi^{\dagger}<180^{\circ}$ in all cases, the analyzed prominence has inverse polarity, i.e., the direction of the prominence magnetic field vector is opposite to the direction expected for a potential field anchored in the photosphere.

In Fig. 9 we show the optical depth and the Doppler width resulting from the inversion of the profiles. The mean optical depth is 0.84 with slightly higher mean values at the prominence feet than at the prominence body. It increases significantly in the spicules. The Doppler width lies between $6 \mathrm{~km} \mathrm{~s}^{-1}$ and $8 \mathrm{~km} \mathrm{~s}^{-1}$ with a mean value of $6.4 \mathrm{~km} \mathrm{~s}^{-1}$. In the same figure we display the inferred LOS velocity. It fluctuates between $\pm 3 \mathrm{~km} \mathrm{~s}^{-1}$. These values are in line with recent Doppler-shift measurements (Schmieder et al. 2010).

Because of the complexity of the He I $1083.0 \mathrm{~nm}$ triplet model and the inversion algorithm, which is based in general optimization methods, it is difficult to give a meaningful estimation
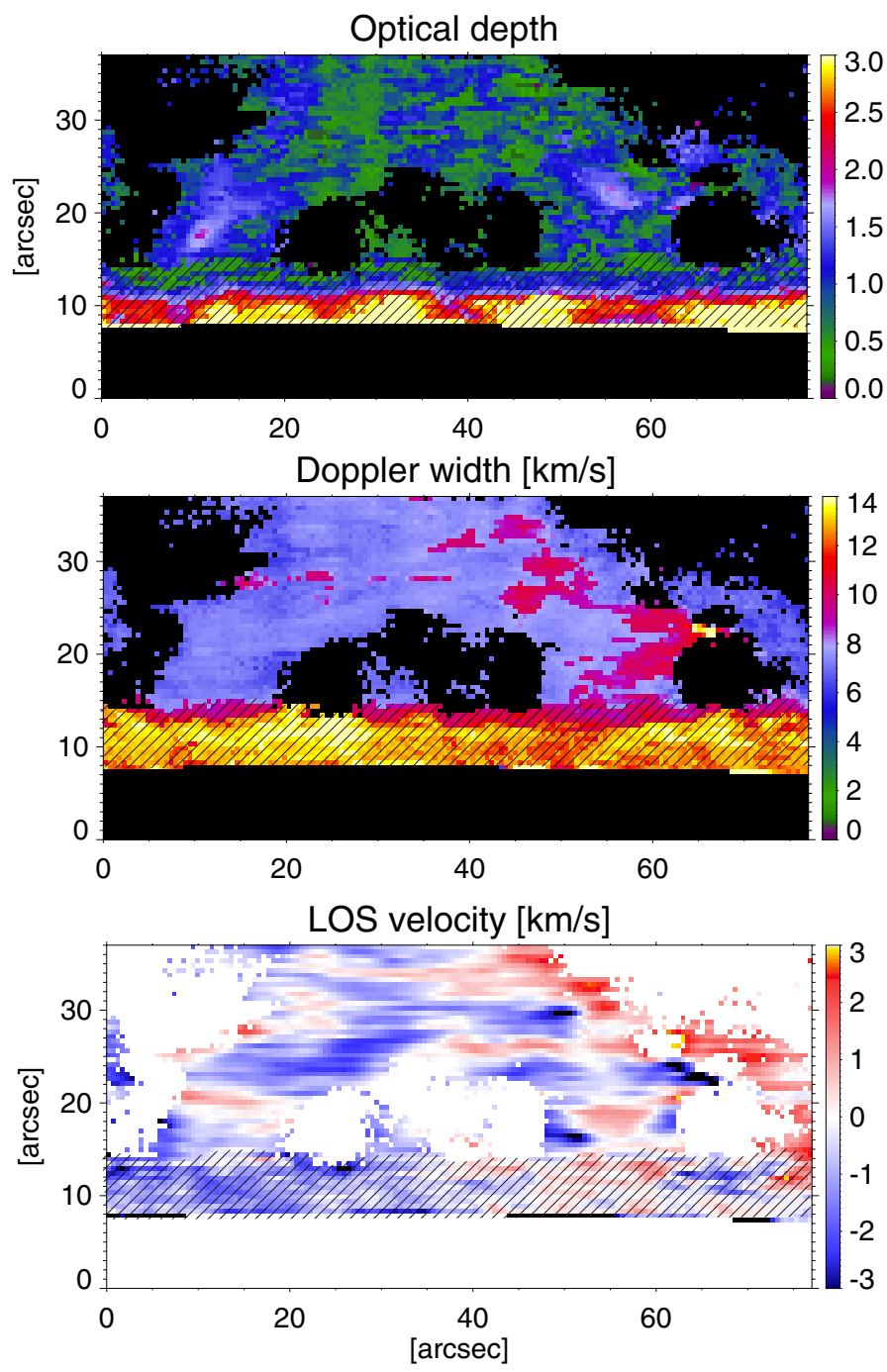

Fig. 9. Optical depth, Doppler width, and line-of-sight velocity inferred with the HAZEL inversion code. The shaded area correspond to solar spicules. As in Fig. 7, the line-shaded areas correspond to the spicules at about $8^{\prime \prime}-14^{\prime \prime}$, excluded in our analysis.

of the statistical errors in the inversion results. In principle, we could use the last iteration of the inversion algorithm (based on a standard Levenberg-Marquardt technique) and estimate the covariance matrix. However, the obtained errors would depend on the exact definition of the merit function itself and would also neglect the effect of degeneracies. Thus, we consider that the way to proceed in the future is to use a fully Bayesian approach. Unfortunately, it has not yet been implemented in HAZEL due to computational constraints.

However, with the merit function we can provide some information about the goodness of the fit. In Fig. 10 we provide the reduced $\chi^{2}$ values resulting from the inversion of the data (see Asensio Ramos et al. 2008). The top and bottom panels stand for the $\chi^{2}$ of the four Stokes parameters and for the quasi-horizontal and quasi-vertical solutions, respectively. First, the $\chi^{2}$ maps are very similar for the two solutions, which means that both solutions are equally probable from an statistical point of view. If we focus on individual panels, it can be seen how the $\chi^{2}$ slightly increases at the boundaries between the prominence and the background, where the signal-to-noise ratio is smaller. There are localized areas where the $\chi^{2}$ increases. For instance, in Stokes $I$, around $x=20^{\prime \prime}$ and $y=25^{\prime \prime}$, there is a local enhancement of 

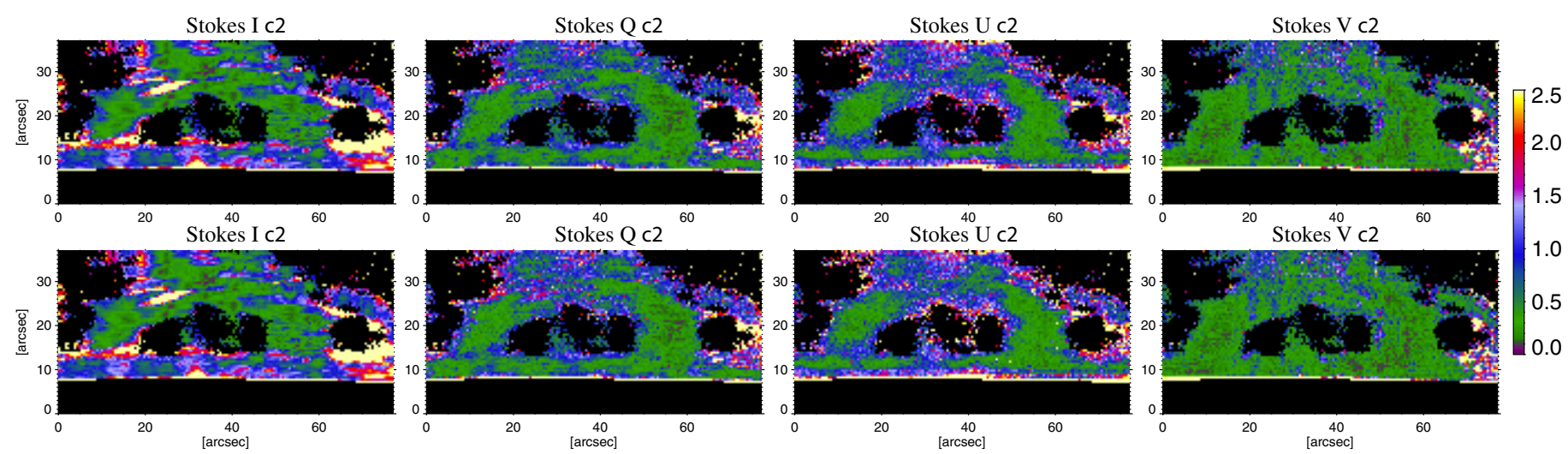

Fig. 10. $\chi^{2}$ values resulting from the inversion of the data. The top panels correspond to one of the quasi-horizontal solutions and the bottom panels to one of the quasi-vertical solutions. As in Fig. 1, the black lower region represent the solar disk. The rest of the dark areas correspond to pixels whose Stokes $Q / I$ or $U / I$ signals did not exceed 5 times their corresponding noise levels.

the $\chi^{2}$ values. This case is due to the presence of a second component in the Stokes I profiles. Overall, the figure shows that the fits are good for most of the pixels.

\section{Summary and conclusions}

In this paper we have shown that spectropolarimetric observations in the He I $1083.0 \mathrm{~nm}$ triplet are very useful for determining the strength and orientation of the magnetic field in solar prominences. In particular, we determined the magnetic field vector in a quiescent solar prominence, using observations of the He I $1083.0 \mathrm{~nm}$ triplet taken with the TIP-II instrument installed at the VTT. Even though the integration time per slit position was on the order of one minute and the total time needed to scan the prominence took $\sim 1.5 \mathrm{~h}$, it is possible to acquire data under stable observing conditions with TIP-II and still maintaining a moderate spatial resolution of $1^{\prime \prime}-11^{\prime \prime} 5$. These integration times are necessary for increasing the signal-to-the-noise ratio and detecting both circular and linear polarization signals in the prominence. These signals are often buried in the noise in "standard" TIP-II observations. The detection of the linear polarization signals allows us to determine the orientation of the field vector while Stokes $V$ is crucial for fixing the field strength.

To infer the field vector we employed the HAZEL inversion code, which includes all necessary atomic physics for interpreting the Stokes $I, Q, U$, and $V$ profiles. We have shown that the use of context data, such as provided by STEREO and SDO, may be crucial for setting up the scattering problem. In our case, STEREO allowed us to determine the prominence heights, its position on the solar disk (viewing angle with respect to the solar vertical), and the orientation of the PIL with respect to the solar limb (or to the LOS).

The He I $1083.0 \mathrm{~nm}$ triplet suffers from two ambiguities: the $90^{\circ}$ ambiguity and the $180^{\circ}$ ambiguity of the Hanle effect. We showed that the observed profiles themselves do not encode sufficient information to solve any of these ambiguities in $90^{\circ}$ scattering geometry (see Fig. 3). Fortunately, from theoretical arguments we can discard two of the solutions. For instance, we can assume that the magnetic field vector belongs to a weakly twisted flux rope or to a sheared arcade that also contains weakly twisted field lines. In this case, the prominence material would be located in dipped magnetic field lines. In these two models, the component of the magnetic field vector along the prominence main axis dominates. Thus, the quasi-vertical solutions, which suggest that the magnetic field vector is almost perpendicular to the prominence main axis can be discarded. The quasi-vertical solution would also require a extremely highly twisted structure, which is rather improbable in prominences (van Ballegooijen $\&$ Martens 1989). Thus, there are two possible solutions only where the magnetic field vector is highly inclined, the quasihorizontal solutions. These two solutions are connected through the $180^{\circ}$ ambiguity of the Hanle effect; i.e., they differ by a $180^{\circ}$ rotation in the plane perpendicular to the LOS.

The quasi-horizontal solutions confirm previous findings about the average field strength in quiescent prominences, which are about $7 \mathrm{G}$, on average. An interesting result is that the field strength seems to be more intense at the prominence feet, reaching values up to $30 \mathrm{G}$ and coinciding with areas where the opacity increases. If the dense plasma is truly suspended in magnetic dips, the existing correlation between the opacity and the field strength may provide additional information for understanding current physical mechanisms for suspending the prominence material. Interestingly, we do not detect any abrupt changes in the prominence field strength contrary to the results of Casini et al. (2003).

Our results for the orientation of the field vector with respect to the solar surface deviate slightly from previous measurements. In particular, we found that the field vector is about $77^{\circ}$ inclined with respect to the solar vertical. This result is between the values reported by, e.g., Leroy (1989) and Bommier et al. (1994), with inclinations of about $60^{\circ}$ from the local vertical, and those reported by Casini et al. (2003), mostly horizontal fields.

Regarding the orientation of the field with respect the prominence main axis, we found it to be $\sim 58^{\circ}$ in one case (dextral chirality) and $\sim 156^{\circ}$ in the corresponding $180^{\circ}$ ambiguous solution (sinistral chirality). The first one differs from previous findings. For instance, Leroy (1989), Bommier et al. (1994), and Casini et al. (2003) report angles below $30^{\circ}$. In contrast, the second solution, $\chi^{\dagger}=156^{\circ}$, implies an acute angle of $24^{\circ}$, in line with previous measurements. In practice, we cannot distinguish between the two of them. We point out that the sinistral chirality case is the most probable solution for southern prominences (Martin 1994), although it may also be possible that the twisting is related to the fact that this prominence was ejected a few hours after the observations. In this case, the more twisted case (dextral) would be the "true" magnetic configuration.

Acknowledgements. We thank M. Collados and M. J. Martínez González for their invaluable help in the TIP-II data reduction. Financial support by the Spanish Ministry of Economy and Competitiveness and the European FEDER 
Fund through project AYA2010-18029 (Solar Magnetism and Astrophysical Spectropolarimetry) is gratefully acknowledged. A.A.R. also acknowledges financial support through the Ramón y Cajal fellowship.

\section{References}

Asensio Ramos, A., \& Trujillo Bueno, J. 2009, in Solar Polarization 5: In Honor of Jan Stenflo, ASP Conf. Ser., 405, 281

Asensio Ramos, A., \& Trujillo Bueno, J. 2010, Mem. Soc. Astron. It., 81, 625

Asensio Ramos, A., Trujillo Bueno, J., \& Landi Degl'Innocenti, E. 2008, ApJ, 683,542

Athay, R. G., Querfeld, C. W., Smartt, R. N., Landi Degl'Innocenti, E., \& Bommier, V. 1983, Sol. Phys., 89, 3

Aulanier, G., \& Demoulin, P. 1998, A\&A, 329, 1125

Aulanier, G., Demoulin, P., van Driel-Gesztelyi, L., Mein, P., \& Deforest, C. 1998, A\&A, 335, 309

Aulanier, G., Démoulin, P., Mein, N., et al. 1999, A\&A, 342, 867

Berger, T. E., Shine, R. A., Slater, G. L., et al. 2008, ApJ, 676, L89

Berger, T. E., Slater, G., Hurlburt, N., et al. 2010, ApJ, 716, 1288

Bommier, V., Landi Degl'Innocenti, E., Leroy, J.-L., \& Sahal-Brechot, S. 1994, Sol. Phys., 154, 231

Casini, R., López Ariste, A., Tomczyk, S., \& Lites, B. W. 2003, ApJ, 598, L67

Casini, R., Bevilacqua, R., \& López Ariste, A. 2005, ApJ, 622, 1265

Casini, R., López Ariste, A., Paletou, F., \& Léger, L. 2009, ApJ, 703, 114

Centeno, R., Trujillo Bueno, J., \& Asensio Ramos, A. 2010, ApJ, 708, 1579

Chae, J. 2010, ApJ, 714, 618

Chae, J., Ahn, K., Lim, E.-K., Choe, G. S., \& Sakurai, T. 2008, ApJ, 689, L73

Collados, M., Lagg, A., Díaz Garcí A, J. J., et al. 2007, The Physics of Chromospheric Plasmas, eds. P. Heinzel, I. Dorotovic̃, \& R. J. Rutten, ASP Conf. Ser., 368, 611

Cox, A. N. 2000, Allen's Astrophysical Quantities, 4th edn. (New York: Springer)

Denker, C., Johannesson, A., Marquette, W., et al. 1999, Sol. Phys., 184, 87

Dudík, J., Aulanier, G., Schmieder, B., Zapiór, M., \& Heinzel, P. 2012, ApJ, 761, 9

Engvold, O. 1976, Sol. Phys., 49, 283

Hillier, A., Berger, T., Isobe, H., \& Shibata, K. 2012a, ApJ, 746, 120

Hillier, A., Hillier, R., \& Tripathi, D. 2012b, ApJ, 761, 106

Hirayama, T. 1985, Sol. Phys., 100, 415

Kaiser, M. L., Kucera, T. A., Davila, J. M., et al. 2008, Space Sci. Rev., 136, 5

Kippenhahn, R., \& Schlüter, A. 1957, ZAp, 43, 36

Labrosse, N., Heinzel, P., Vial, J.-C., et al. 2010, Space Sci. Rev., 151, 243

Landi Degl'Innocenti, E., \& Landolfi, M. 2004, Astrophys. Space Sci. Lib., 307

Lemen, J. R., Title, A. M., Akin, D. J., et al. 2012, Sol. Phys., 275, 17

Leroy, J. L. 1989, in Dynamics and Structure of Quiescent Solar Prominences (Dordrecht: Kluwer Academic Publisher), Proc. Workshop, 150, 77

Leroy, J. L., Bommier, V., \& Sahal-Brechot, S. 1983, Sol. Phys., 83, 135

Lin, H., Penn, M. J., \& Kuhn, J. R. 1998, ApJ, 493, 978

López Ariste, A., \& Aulanier, G. 2007, in The Physics of Chromospheric

Plasmas, ASP Conf. Ser., 368, 291
Low, B. C., \& Petrie, G. J. D. 2005, ApJ, 626, 551

Mackay, D. H., Karpen, J. T., Ballester, J. L., Schmieder, B., \& Aulanier, G. 2010, Space Sci. Rev., 151, 333

Martin, S. F. 1998, New Perspectives on Solar Prominences, IAU Colloq. 167, ASP Conf. Ser., 150, 419

Martin, S. F., Bilimoria, R., \& Tracadas, P. W. 1994, in Solar Surface Magnetism, NATO ASI Series C433, eds. R. J. Rutten, \& C. J. Schrijver (Dordrecht: Kluwer Academic Publishers), 303

Martínez González, M. J., Asensio Ramos, A., Manso Sainz, R., Beck, C., \& Belluzzi, L. 2012, ApJ, 759, 16

Merenda, L., Trujillo Bueno, J., Landi Degl'Innocenti, E., \& Collados, M. 2006, ApJ, 642, 554

Merenda, L., Trujillo Bueno, J., \& Collados, M. 2007, The Physics of Chromospheric Plasmas, ASP Conf. Ser., 368, 347

Okamoto, T. J., Tsuneta, S., Berger, T. E., et al. 2007, Science, 318, 1577

Orozco Suárez, D., Asensio Ramos, A., \& Trujillo Bueno, J. 2012, ApJ, 761, L25

Orozco Suárez, D., Asensio Ramos, A., \& Trujillo Bueno, J. 2013, Highlights of Spanish Astrophysics VII, Proc. of the X Scientific Metting of SEA, eds. J. C. Guirado, L. M., Lara, V. Guilis, \& J. Grorgas, 786

Pécseli, H., \& Engvold, O. 2000, Sol. Phys., 194, 73

Pesnell, W. D., Thompson, B. J., \& Chamberlin, P. C. 2012, Sol. Phys., 275, 3

Pettit, E. 1943, ApJ, 98, 6

Plunkett, S. P., Vourlidas, A., Šimberová, S., et al. 2000, Sol. Phys., 194, 371

Querfeld, C. W., Smartt, R. N., Bommier, V., Landi Degl'Innocenti, E., \& House, L. L. 1985, Sol. Phys., 96, 277

Ramelli, R., Trujillo Bueno, J., Bianda, M., \& Asensio Ramos, A. 2011, Solar Polarization 6, eds. J. R. Kuhn, D. M. Havoington, H. Lin, et al., ASP Conf., 437, 109

Régnier, S., Walsh, R. W., \& Alexander, C. E. 2011, A\&A, 533, L1

Rust, D. M., \& Kumar, A. 1994, Sol. Phys., 155, 69

Schad, T. A., Penn, M. J., \& Lin, H. 2013, ApJ, 768, 111

Schmieder, B., Chandra, R., Berlicki, A., \& Mein, P. 2010, A\&A, 514, A68

Tandberg-Hanssen, E. 1995, Astrophys. Space Sci. Lib., 199,

Tandberg-Hanssen, E. 1998, New Perspectives on Solar Prominences, IAU Colloq. 167, ASP Conf. Ser., 150, 11

Tandberg-Hanssen, E. 2011, Sol. Phys., 269, 237

Tandberg-Hanssen, E., \& Anzer, U. 1970, Sol. Phys., 15, 158

Trujillo Bueno, J. 2010, in Magnetic Coupling between the Interior and Atmosphere of the Sun (Springer Verlag), 118

Trujillo Bueno, J., \& Asensio Ramos, A. 2007, ApJ, 655, 642

Trujillo Bueno, J., \& Manso Sainz, R. 2002, Nuovo Cimento C, Geophysics Space Physics, 25, 783

Trujillo Bueno, J., Landi Degl'Innocenti, E., Collados, M., Merenda, L., \& Manso Sainz, R. 2002, Nature, 415, 403

Trujillo Bueno, J., Merenda, L., Centeno, R., Collados, M., \& Landi Degl'Innocenti, E. 2005, ApJ, 619, L191

van Ballegooijen, A. A., \& Martens, P. C. H. 1989, ApJ, 343, 971

van Ballegooijen, A. A., \& Cranmer, S. R. 2010, ApJ, 711, 164

Zirker, J. B., Engvold, O., \& Yi, Z. 1994, Sol. Phys., 150, 81 